\title{
A tale of two arteries: dual posterior cerebral arteries with vascular bridges. A possible protective pattern?
}

\author{
Y. Mansour ${ }^{1,2}$, R. Kulesza ${ }^{1}$ \\ ${ }^{1}$ Department of Anatomy, Lake Erie College of Osteopathic Medicine, Erie, PA, United States \\ 2Department of Otolaryngology and Facial Plastic Surgery, Henry Ford Macomb, Clinton Twp, MI, United States
}

[Received: 19 June 2020; Accepted: 1 July 2020]

Stroke is a common morbidity and a frequent cause of disability and even death. The impact of cerebrovascular events is dictated by the brain region involved and can be complicated by anatomical variations. One of the most common variations impacting the cerebral vasculature is the presence of a foetal posterior cerebral artery. This vessel arises from the internal carotid artery instead of the basilar artery and is often associated with more extensive injury in cerebrovascular events. Herein, we report the case of a 60-year-old male who had numerous arterial abnormalities, including a kink and a coil of the left internal carotid, two posterior communicating arteries on the right and two posterior cerebral arteries (PCA) on the left, one arising from the internal carotid (foetal PCA) and one from the basilar. The foetal PCA supplied the thalamus, splenium of the corpus callosum and primary visual cortex. The basilar PCA supplied the midbrain and parts of the occipital lobe. These PCA were connected to each other by a vascular bridge and the foetal PCA was connected to the middle cerebral artery by an additional vascular bridge. This vascular pattern would appear to provide collateral support around blockages in the internal carotid and main stem middle and PCA. (Folia Morphol 2021; 80, 2: 455-459)

Key words: variation, cerebral, vasculature, foetal

\section{INTRODUCTION}

Stroke is a leading cause of death and a major cause of disability [6]. Stroke, its treatment and subsequent mortality can be complicated by variations in arterial patterns. One of the most common intracranial arterial variations is persistence of the foetal posterior communicating artery (PCOM), which occurs in approximately $20 \%$ of subjects $[5,7,8,10]$. Therefore, clinical knowledge of both normal and variant cerebrovascular patterns is important in predicting vascular risk, recognising common and atypical stroke syndromes and planning neurointerventions $[2,10,11]$.
Herein, we report a rare case of a subject with two posterior cerebral arteries (PCA) on the left and two PCOMs on the right side. Additionally, this subject had two abnormalities involving his left internal carotid artery [1, 4]. We discuss the embryological origin of these vessels and their potential impacts/ /consequences.

\section{CASE REPORT}

\section{Case presentation}

A 60-year-old male presented to the emergency department with the chief complaints of seeing 


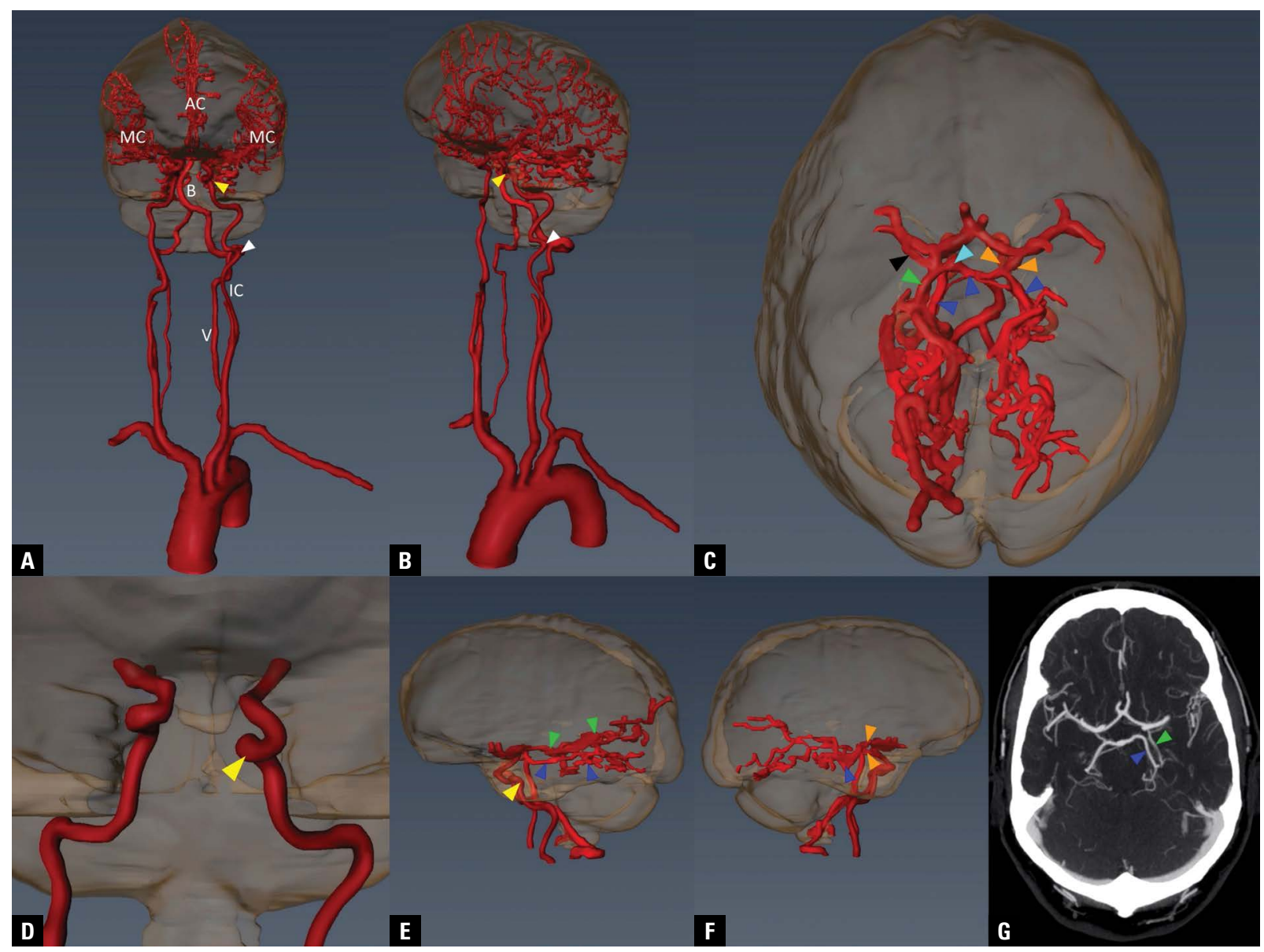

Figure 1. Images of three-dimensional reconstruction $(\mathbf{A}-\mathbf{F})$ and computed tomography angiography $(\mathbf{G})$ of this patient's arterial pattern. The views in panels $\mathbf{A}$ and $\mathbf{B}$ show the carotid and vertebral arteries (V) from their origin in the neck. The formation of the basilar artery (B) appeared normal. In the neck, the left internal carotid (IC) artery (ICA) had both a coil (yellow arrowhead; observed also in panel D) and a kink (white arrowhead). The ICAs were found to give rise to the middle (MC) and anterior (AC) cerebral arteries. In panels $\mathbf{C}, \mathbf{E}$ and $\mathbf{F}$ the abnormal posterior cerebral arteries (PCA) and posterior communicating artery (PCOM) are identified. Panel $\mathbf{C}$ shows a top-down view. The blue arrowheads indicate the basilar PCA [bPCA], and the orange arrowhead indicates the PCOM. On the patient's right side there were two PCOMs. On the patients left, there were two PCAs: one from the ICA (foetal PCA [fPCA]) and one from the basilar (bPCA). There were two vascular bridges on the left: one from the fPCA to the middle cerebral artery (black arrowhead) and one from the PPCA to the bPCA. Panel E shows the two PCAs from the patients left side. Panel $\mathbf{F}$ shows the double PCOM from the patient's right side. Panel $\mathbf{G}$ shows a slice from the computed tomography angiography study. The PPCA is indicated by the green arrowhead and the bPCA is indicated by the blue arrowhead.

flashing lights in his right eye and left arm weakness. His neurological exam was non-focal. He underwent computed tomography (CT) and computed tomography angiography (CTA) imaging to rule out possible stroke. The CT series was imported into Amira (Version 6.7.0) and a slice by slice approach was taken to create a three-dimensional (3D) rendering of both his carotid and vertebral circulations.

\section{Imaging findings}

Examination of the 3D model (Fig. 1A, B) of this subject's cerebral vasculature revealed multiple variations. On the left internal carotid artery (ICA) there was a cervical kink (Fig. 1, white arrowhead) and a cavernous coil (Fig. 1, yellow arrowhead) [2]. On the left side there was a PCA that arose at the basilar bifurcation ("basilar PCA" [bPCA]; Fig. 1, blue arrowheads) and a foetal PCA (fPCA; Fig. 1, green arrowheads) that continued directly from the ICA. The CT and 3D reconstruction clearly showed both of these arteries wrapping around the midbrain and distributing within the occipital lobe. The fPCA had a vascular bridge to the middle cerebral artery (MCA; Fig. 1, black arrowhead) and bPCA (Fig. 1, light blue arrowhead). On the right side there were two PCOMs (Fig. 1, orange arrowheads). A video of the 3D reconstruction can be found here: https://drive.google.com/file/d/1AfJI9WwRe4LNhH2gNECcjPqQa75hRtiY/view?usp=sharing. 


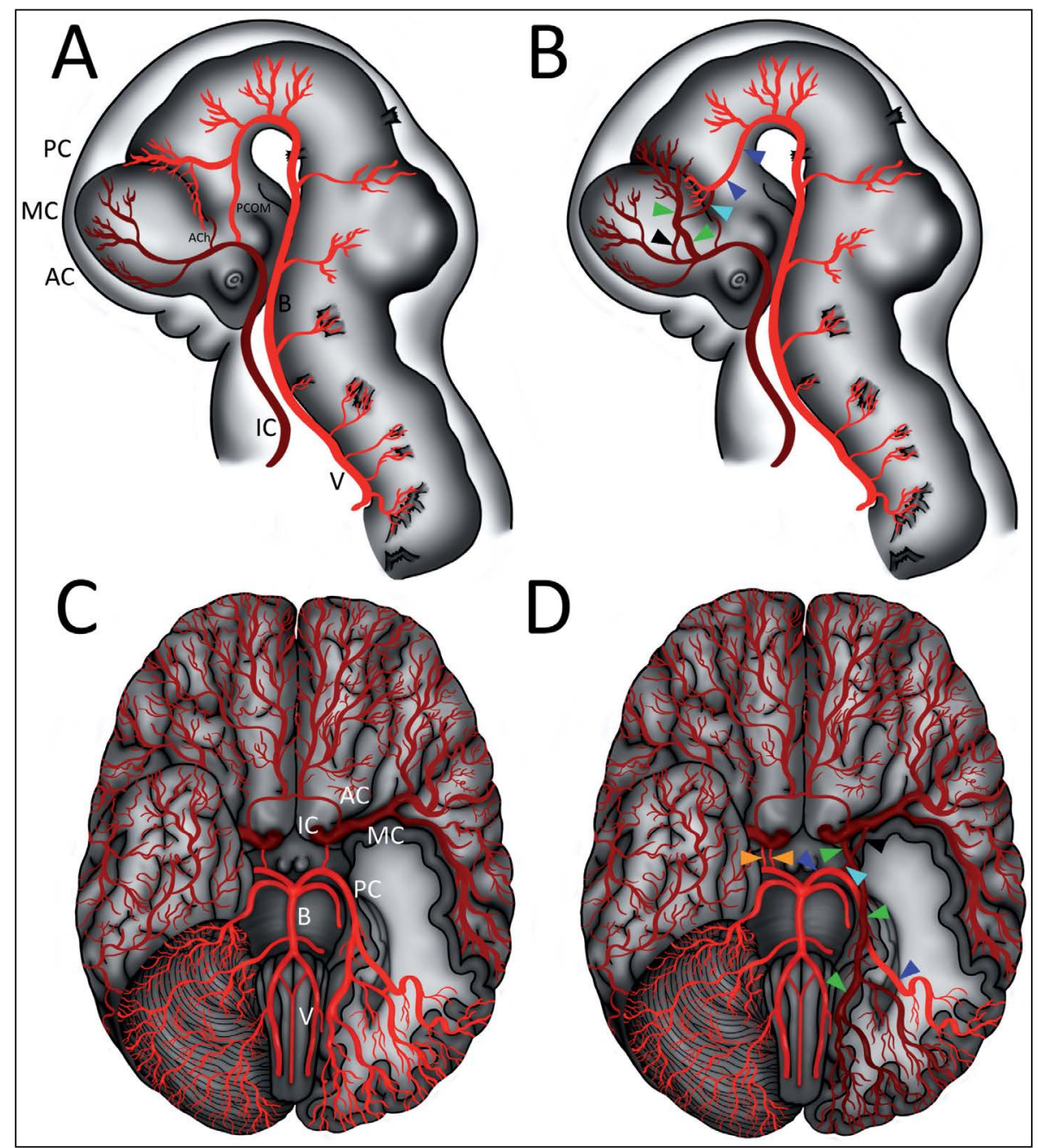

Figure 2. Proposed arterial patterns. Shown in panel $\mathbf{A}$ is the typical embryologic pattern that forms the posterior communicating artery (PCOM) and posterior cerebral arteries (PCA) and the pattern we propose developed in this subject in panel $\mathbf{B}$. Shown in panel $\mathbf{C}$ is the typical arterial pattern and in panel $\mathbf{D}$ is the arterial pattern discovered in this case study. Key to arrowheads: green — foetal PCA, blue — basilar PCA, orange — PCOM, black — foetal PCA to middle cerebral artery vascular bridge, light blue — foetal PCA to basilar PCA vascular bridge; AC — anterior cerebral arteries; ACh — anterior choroidal; B — basilar artery; IC — internal carotid; MC — middle cerebral arteries; PC — posterior cerebral arteries; V - vertebral arteries.

\section{Correlative anatomy}

Developmentally, the arterial supply of the brain is derived from vertebral and ICAs and the PCOM initially connects these two circulations (Fig. 2A). The PCA is normally derived from the PCOM and supplies the midbrain, diencephalon and caudal telencephalon. We propose that the arterial variations identified in this subject arose from the altered embryonic vascular pattern shown schematically in Figure 2B. Careful study of the 3D reconstruction and CTA series allowed us to develop a vascular map (Fig. 2C, D). In the CTA, we carefully traced branches from both the bPCA and FPCA and constructed maps of their vascular territories. In this subject, the BPCA supplied the central aspect of the midbrain and the lateral aspect of the
PCA territory (Fig. 3). The PPCA supplied the lateral aspect of the midbrain, the entire thalamus, splenium of the corpus callosum and the medial aspect of the occipital lobe including the calcarine sulcus.

\section{DISCUSSION}

The PCA normally develops as a direct continuation of the embryonic PCOM and supplies the midbrain, thalamus and occipital lobe. The most common variant of the PCOM/PCA is an PPCA where this vessel forms as a direct branch from the ICA $[5,7,8,10]$. When an FPCA is present, the ICA provides arterial supply to the entire cerebral hemisphere, thalamus and midbrain via anterior, middle and posterior cerebral arteries. Accordingly, if a subject with an fPCA 

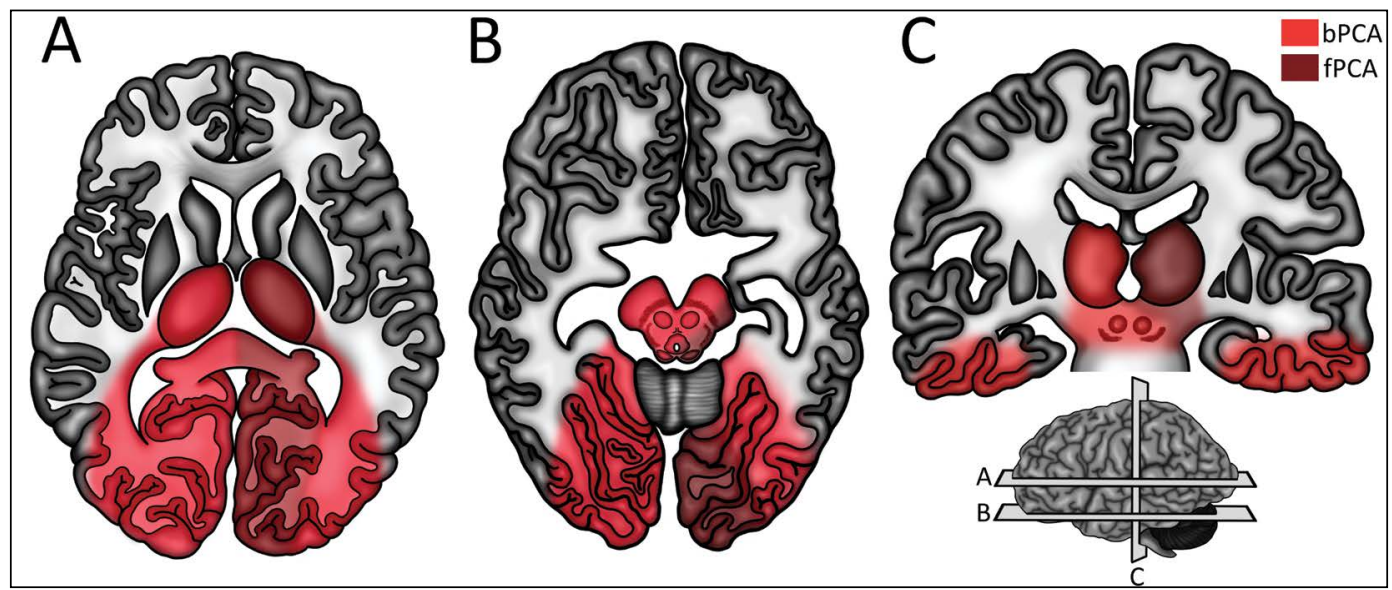

Figure 3. Shown in panels $\mathbf{A}$ and $\mathbf{B}$ are transverse slices through the brain at the level of the thalamus (A) and midbrain (B). Shown in panel C is a coronal slice through the brain at the level of the thalamus. On the left side of each image (the patient's right), the territory of the basilar posterior cerebral arteries (bPCA) is shown in red. On the right side of each image (the patient's left) the territory of both the bPCA and foetal posterior cerebral arteries (fPCA) are shown. Note that in the subject of this case study, the fPCA supplies the lateral and posterior midbrain, thalamus, splenium of the corpus callosum and primary visual cortex. The inset in the bottom right shows the approximate levels and locations of the slices shown in panels A-C.

has a blockage of the ICA, this can impair blood flow to the entire thalamus and cerebral hemisphere with often catastrophic results [5, 9]. However, the presence of an PPCA is not considered an additional risk factor for stroke [3].

The subject of this case presentation has both a bPCA and an FPCA and these vessels made major connections to other vessels in the left hemisphere through vascular bridges. In our 3D reconstruction and study of the CTA series, we found the PPCA and bPCA were connected by a small vascular bridge near their origin [8], but there were no additional connections between the two PCAs. Further, we found an additional vascular bridge connecting the PPCA and MCA. Together, we believe this arterial architecture may provide a protective support pattern. Specifically, if the subject of this case presentation were to have a thrombus in the ICA or in the main stem of PPCA, bPCA or MCA on the left, the bridging vessels could provide collateral support and potentially prevent ischaemia or infarct in PCA or MCA territories.

\section{CONCLUSIONS}

The fPCA is a fairly common anatomical variation involving arterial support of the midbrain, thalamus and occipital lobe. Vascular compromise involving the anterior circulation in a subject with an PPCA is often associated with extensive ischaemia and poor outcomes. However, the presence of vascular bridges connecting the fPCA to surrounding main-stem vessels may provide protective anastomoses.

\section{Acknowledgements}

The authors would like to thank the University of Pittsburgh Medical Centre Hamot, Department of Neurology for the CT and CTA data.

\section{REFERENCES}

1. Benson JC, Brinjikji W, Messina SA, et al. Cervical internal carotid artery tortuosity: A morphologic analysis of patients with acute ischemic stroke. Interv Neuroradiol. 2020; 26(2): 216-221, doi: 10.1177/1591019919891295, indexed in Pubmed: 31766962.

2. Brzegowy P, Polak J, Wnuk J, et al. Middle cerebral artery anatomical variations and aneurysms: a retrospective study based on computed tomography angiography findings. Folia Morphol. 2018; 77(3): 434-440, doi: 10.5603/ FM.a2017.0112, indexed in Pubmed: 29235088.

3. de Monyé $C$, Dippel DWJ, Siepman TAM, et al. Is a fetal origin of the posterior cerebral artery a risk factor for TIA or ischemic stroke? A study with 16-multidetector-row CT angiography. J Neurol. 2008; 255(2): 239-245, doi: 10.1007/s00415-008-0699-8, indexed in Pubmed: 18274809.

4. Griessenauer CJ, Yalcin B, Matusz P, et al. Analysis of the tortuosity of the internal carotid artery in the cavernous sinus. Childs Nerv Syst. 2015; 31(6): 941-944, doi: 10.1007/

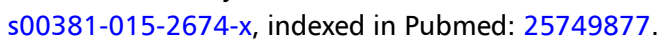

5. Klimek-Piotrowska W, Kopeć M, Kochana M, et al. Configurations of the circle of Willis: a computed tomography angiography based study on a Polish population. Folia Morphol. 2013; 72(4): 293-299, doi: 10.5603/ fm.2013.0049, indexed in Pubmed: 24402749. 
6. Stroke. Centers for Disease Control and Prevention. https:// www.cdc.gov/stroke/index (2020, April 7).

7. Uchino A, Saito N, Takahashi M, et al. Variations of the posterior cerebral artery diagnosed by MR angiography at 3 tesla. Neuroradiology. 2016; 58(2): 141-146, doi: 10.1007/ s00234-015-1614-5, indexed in Pubmed: 26553301.

8. Vasović L, Trandafilović M, Jovanović I, et al. An excess vessel in the posterior part of the human cerebral arterial circle (CAC): a case series. BMC Neurol. 2010; 10: 53, doi: 10.1186/1471-2377-10-53, indexed in Pubmed: 20573186.

9. Yu J, Qu L, Xu B, et al. Current understanding of dolichoarteriopathies of the internal carotid artery: a review. Int
J Med Sci. 2017; 14(8): 772-784, doi: 10.7150/ijms.19229, indexed in Pubmed: 28824313.

10. Zampakis $P$, Panagiotopoulos V, Petsas $T$, et al. Common and uncommon intracranial arterial anatomic variations in multi-detector computed tomography angiography (MDCTA). What radiologists should be aware of. Insights Imaging. 2015; 6(1): 33-42, doi: 10.1007/s13244-0140381-x, indexed in Pubmed: 25680324.

11. Zurada A, Gielecki JS. A novel formula for the classification of blood vessels according to symmetry, asymmetry and hypoplasia. Folia Morphol. 2007; 66(4): 339-345, indexed in Pubmed: 18058758. 